\title{
Fixed points in modular spaces with new type contractivity
}

\author{
T.L. Shateri \\ Hakim Sabzevari University \\ Email: t.shateri@hsu.ac.ir
}

Copyright (C2014 T.L. Shateri. This is an open access article distributed under the Creative Commons Attribution License, which permits unrestricted use, distribution, and reproduction in any medium, provided the original work is properly cited.

\begin{abstract}
In this present paper, we prove a common fixed point theorem for self maps in modular spaces. Also one corollary, which shows that our main theorem is generalized version of the main theorem of [A. Razani, E. Nabizadeh, M. Beyg Mohamadi and S. Homaei Pour, Abs. Appl. Anal. 2007, Article ID 40575] is given.
\end{abstract}

Keywords: Fixed point, contraction, modular, modular space.

\section{Introduction}

The theory of modular spaces was introduced by Nakano [1] in 1950 and generalized by Musielak and Orlicz [2], Koshi and Shimogaki [3] and Yamamuro [4] and their collaborators. The monographic exposition of the theory of Orlicz spaces may be found in in the book of Krasnosel'skii and Rutickii[5]. We referred the reader for the theory of Orlicz spaces and modular spaces, to the books [6,7]. Fixed point theorems in modular spaces, generalizing the classical Banach fixed point theorem in metric spaces, have been studied extensively by many mathematicians such as Arandelović, [8], Edelstein [9], Ćirić [10], Rakotch [11], Reich [12], Kirk [13]. In addition, Razani et al. [14] proved some fixed point theorems of non linear and asymptotic contractions in modular spaces. Also, quasi-contraction mappings in modular spaces without $\Delta_{2}$-condition were investigated by Khamsi [15]. Kuaket and Kumam [16] proved the existence of fixed points of asymptotic pointwise contractions in modular spaces. Moreover Chen and Wang [17] proved the fixed points of asymptotic pointwise nonexpansive mappings in modular spaces.

In this paper we establish a fixed point theorem for self maps in modular spaces with new type contractivity.

Definition 1.1 Let $\mathcal{X}$ be an arbitrary vector space over $\mathbb{F}(=\mathbb{R}$ or $\mathbb{C})$.

A functional $\rho: \mathcal{X} \rightarrow[0, \infty]$ is called modular if for all $x, y \in \mathcal{X}$,

(i) $\rho(x)=0$ if and only if $x=0$,

(ii) $\rho(\alpha x)=\rho(x)$ for every $\alpha \in \mathbb{F}$ with $|\alpha|=1$,

(iii) $\rho(\alpha x+\beta y) \leq \rho(x)+\rho(y)$ if $\alpha, \beta \geq 0$ and $\alpha+\beta=1$.

Definition 1.2 If (iii) in definition 1.1 is replaced by

$$
\rho(\alpha x+\beta y) \leq \alpha^{s} \rho(x)+\beta^{s} \rho(y),
$$

for $\alpha, \beta \geq 0, \alpha+\beta=1$ with an $s \in(0,1]$, then we say that $\rho$ is an $s$-convex modular, and if $s=1, \rho$ is called a convex modular. 
A modular $\rho$ defines a corresponding modular space, i.e., the vector space $\mathcal{X}_{\rho}$ given by to

$\mathcal{X}_{\rho}=\{x \in \mathcal{X}: \quad \rho(\lambda x) \rightarrow 0$ as $\lambda \rightarrow 0\}$.Let $\rho$ be a convex modular, the modular space $\mathcal{X}_{\rho}$ can be equipped with a norm called the Luxemburg norm, defined by

$$
\|x\|_{\rho}=\inf \left\{\lambda>0 \quad ; \quad \rho\left(\frac{x}{\lambda}\right) \leq 1\right\} .
$$

Definition 1.3 Let $\mathcal{X}_{\rho}$ be a modular space and let $\left\{x_{n}\right\}$ and $x$ be in $\mathcal{X}_{\rho}$. Then

(i) $\left\{x_{n}\right\}$ is said to be $\rho$-convergent to $x$ and write $x_{n} \stackrel{\rho}{\longrightarrow} x$ if $\rho\left(x_{n}-x\right) \rightarrow 0$ as $n \rightarrow \infty$.

(ii) $\left\{x_{n}\right\}$ is called $\rho$-Cauchy if $\rho\left(x_{n}-x_{m}\right) \rightarrow 0$ as $n, m \rightarrow \infty$.

(iii) A subset $\mathcal{S}$ of $\mathcal{X}_{\rho}$ is called $\rho$-complete complete if any $\rho$-Cauchy sequence is $\rho$-convergent to an element of $\mathcal{S}$.

(iv) A subset $B$ of $\mathcal{X}_{\rho}$ is called $\rho$-closed if for any sequence $\left\{x_{n}\right\} \subseteq B$ with $x_{n} \stackrel{\rho}{\longrightarrow} x$, we have $x \in B$.

(v) We say the modular $\rho$ has the Fatou property if $\rho(x) \leq \liminf _{n \rightarrow \infty} \rho\left(x_{n}\right)$ whenever $x_{n} \stackrel{\rho}{\longrightarrow} x$.

(vi) $\rho$ is said to satisfy the $\Delta_{2}$-condition if $\rho\left(2 x_{n}\right) \rightarrow 0$ whenever $\rho\left(x_{n}\right) \rightarrow 0$ as $n \rightarrow \infty$.

Remark 1.4 Note that, if $x \in \mathcal{X}_{\rho}$ then $\rho(a x)$ is an increasing function of $a>0$. Suppose $0<a<b$, then property (iii) of Definition 1.1 with $y=0$ shows that $\rho(a x)=\rho\left(\frac{a}{b} b x\right) \leq \rho(b x)$ for all $x \in \mathcal{X}$. Moreover, if $\rho$ is a convex modular on $\mathcal{X}$ and $|\alpha| \leq 1$, then $\rho(\alpha x) \leq \alpha \rho(x)$ and also $\rho(x) \leq \frac{1}{2} \rho(2 x)$ for all $x \in \mathcal{X}$.

Definition 1.5 A function $T: \mathcal{X}_{\rho} \rightarrow \mathcal{X}_{\rho}$ is called $\rho$-continuous if

$\rho\left(x_{n}-x\right) \rightarrow 0$, then $\rho\left(T\left(x_{n}\right)-T(x)\right) \rightarrow 0$.

\section{Main results}

Throughout this paper, we assume that the modular $\rho$ satisfies the $\Delta_{2}$-condition. In this section, by using some ideas from $[14,18]$ we will prove a fixed point theorem for a new type of contractivity as follows.

Theorem 2.1 Let $\mathcal{X}_{\rho}$ be a $\rho$-complete modular space, where $\rho$ satisfies the $\Delta_{2}$-condition. Suppose that $\varphi: \mathbb{R}^{+} \rightarrow$ $[0, \infty)$ is an increasing and upper semicontinuous function satisfying

$\varphi(t)<t, \quad(t>0)$.

Let $C$ be a $\rho$-closed subset of $\mathcal{X}_{\rho}$ and let $T, S: C \rightarrow C$ be mappings such that there exist $\alpha, \beta \in \mathbb{R}^{+}$with $\alpha>\beta$, and

$\rho(\alpha(T x-S y)) \leq \varphi(\rho(\beta(x-y)))$,

for all $x, y \in C$. Then $T$ and $S$ have a unique common fixed point in $C$.

First we prove that any fixed point of $T$ is also a fixed point of $S$, and conversely. Suppose $T x=x$, hence we have from (4)

$0 \leq \rho(\alpha(x-S x)) \leq \varphi(\rho(\beta(x-x)))=0$,

since $\alpha>0$, so $S x=x$. Similarly, if $S x=x$, then $T x=x$.

Now, we prove that if $T$ and $S$ have a common fixed point, then the fixed point is unique. Let $T x=S x=x$ and $T y=S y=y$. If $x \neq y$, then (4) implies that

$\rho(\beta(x-y))<\rho(\alpha(x-y))=\rho(\alpha(T x-S y)) \leq \varphi(\rho(\beta(x-y)))$,

which is a contradiction. Therefore $x=y$.

Suppose $x_{0} \in C$ and put $x_{2 n+1}=T x_{2 n}, x_{2 n+2}=S x_{2 n+1}$ for all $n=0,1,2, \cdots$. We may suppose that for any $n, x_{n+1} \neq x_{n}$, otherwise $T$ or $S$ has a fixed point and the proof is complete. Now, we have

$$
\begin{aligned}
\rho\left(\alpha\left(x_{2 n+1}-x_{2 n}\right)\right) & =\rho\left(\alpha\left(T x_{2 n}-S x_{2 n-1}\right)\right) \leq \varphi\left(\rho\left(\beta\left(x_{2 n}-x_{2 n-1}\right)\right)\right) \\
& <\rho\left(\beta\left(x_{2 n}-x_{2 n-1}\right)\right),
\end{aligned}
$$


similarly

$$
\begin{aligned}
\rho\left(\alpha\left(x_{2 n+2}-x_{2 n+1}\right)\right) & =\rho\left(\alpha\left(S x_{2 n+1}-T x_{2 n}\right)\right) \leq \varphi\left(\rho\left(\beta\left(x_{2 n+1}-x_{2 n}\right)\right)\right) \\
& <\rho\left(\beta\left(x_{2 n+1}-x_{2 n}\right)\right) .
\end{aligned}
$$

Hence (7) and (8) imply that

$\rho\left(\alpha\left(x_{n+1}-x_{n}\right)\right) \leq \varphi\left(\rho\left(\beta\left(x_{n}-x_{n-1}\right)\right)\right)<\rho\left(\beta\left(x_{n}-x_{n-1}\right)\right)(n \geq 1)$.

Consequently, $\left\{\rho\left(\alpha\left(x_{n+1}-x_{n}\right)\right)\right\}$ is decreasing and bounded from below. Hence

$\left\{\rho\left(\alpha\left(x_{n+1}-x_{n}\right)\right)\right\}$ converges to $z$. Now, if $z \neq 0$,

$$
\begin{aligned}
z=\lim _{n \rightarrow \infty} \rho\left(\alpha\left(x_{n+1}-x_{n}\right)\right) & \leq \lim _{n \rightarrow \infty} \varphi\left(\rho\left(\beta\left(x_{n}-x_{n-1}\right)\right)\right) \\
& <\lim _{n \rightarrow \infty} \varphi\left(\rho\left(\alpha\left(x_{n}-x_{n-1}\right)\right)\right)=\varphi(z),
\end{aligned}
$$

which is a contradiction, hence $z=0$.

Now, we show that $\left\{x_{n}\right\}$ is a $\rho$-cauchy sequence in $\mathcal{X}_{\rho}$. If $\left\{\beta x_{n}\right\}$ is not a $\rho$-cauchy sequence, then there exists $\varepsilon>0$ and sequences $\left\{m_{k}\right\},\left\{n_{k}\right\}$ of integers with $m_{k}>n_{k} \geq k$ and

$\rho\left(\beta\left(x_{m_{k}}-x_{n_{k}}\right)\right) \geq \varepsilon \quad(k \in \mathbb{N})$.

Moreover, corresponding to odd numbers $n_{k}$, we can choose even numbers $m_{k}$ in such a way that it is the smallest integer with $m_{k}>n_{k}$ such that

$\rho\left(\beta\left(x_{m_{k-2}}-x_{n_{k}}\right)\right)<\varepsilon$.

In fact, let $m_{k}$ be the smallest even number exceeding $n_{k}$ for which (10) holds, and

$$
N_{k}=\left\{m \in \mathbb{N}_{e} \mid \exists n_{k} \in \mathbb{N}_{o} ; \rho\left(\beta\left(x_{m}-x_{n_{k}}\right)\right) \geq \varepsilon, m>n_{k} \geq k\right\} .
$$

It is clear that $N_{k} \neq \emptyset$ and by well ordering principle, the minimum element of $N_{k}$ exists and is denoted by $m_{k}$, and clearly (11) holds.

Now, let $\alpha_{0} \in \mathbb{R}^{+}$be such that $\frac{\beta}{\alpha}+\frac{1}{\alpha_{0}}=1$, then we have

$$
\begin{aligned}
\rho\left(\beta\left(x_{m_{k}}-x_{n_{k}}\right)\right) & =\rho\left(\frac{\beta \alpha}{\alpha}\left(\alpha\left(x_{m_{k}}-x_{n_{k+2}}\right)\right)+\frac{1}{\alpha_{0}}\left(\alpha_{0} \beta\left(x_{n_{k+2}}-x_{n_{k}}\right)\right)\right) \\
& \leq \rho\left(\alpha\left(x_{m_{k}}-x_{n_{k+2}}\right)\right)+\rho\left(\alpha_{0} \beta\left(x_{n_{k+2}}-x_{n_{k}}\right)\right) \\
& \leq \varphi\left(\rho\left(\beta\left(x_{m_{k-1}}-x_{n_{k+1}}\right)\right)+\rho\left(\alpha_{0} \beta\left(x_{n_{k+2}}-x_{n_{k}}\right)\right)\right. \\
& <\varepsilon+\rho\left(\alpha_{0} \beta\left(x_{n_{k+2}}-x_{n_{k}}\right)\right) .
\end{aligned}
$$

If $k \rightarrow \infty$, by $\Delta_{2}$-condition, $\rho\left(\alpha_{0} \beta\left(x_{n_{k+2}}-x_{n_{k}}\right)\right) \rightarrow 0$, hence $\lim _{k \rightarrow \infty} \rho\left(\beta\left(x_{m_{k}}-x_{n_{k}}\right)\right)=\varepsilon$. Therefore,

$$
\begin{aligned}
\rho\left(\beta\left(x_{m_{k}}-x_{n_{k}}\right)\right) & \leq \rho\left(\alpha\left(x_{m_{k+1}}-x_{n_{k+1}}\right)\right)+\rho\left(2 \alpha_{0} \beta\left(x_{m_{k}}-x_{m_{k+1}}\right)\right)+\rho\left(2 \alpha_{0} \beta\left(x_{n_{k+1}}-x_{n_{k}}\right)\right) \\
& \leq \varphi\left(\rho\left(\beta\left(x_{m_{k}}-x_{n_{k}}\right)\right)\right)+\rho\left(2 \alpha_{0} \beta\left(x_{m_{k}}-x_{m_{k+1}}\right)\right)+\rho\left(2 \alpha_{0} \beta\left(x_{n_{k+1}}-x_{n_{k}}\right)\right) .
\end{aligned}
$$

Therefore, as $k \rightarrow \infty$, we get $\varepsilon \leq \varphi(\varepsilon)$, which is a contradiction. Hence $\left\{\beta x_{n}\right\}$ is a $\rho$-cauchy sequence, and by $\Delta_{2}$-condition, $\left\{x_{n}\right\}$ is a $\rho$-cauchy sequence. Since $\mathcal{X}_{\rho}$ is complete, there is a $w \in C$ such that $\rho\left(x_{n}-w\right) \rightarrow 0$, as $n \rightarrow \infty$. Now, we show that $w$ is the common fixed point of $T$ and $S$. Put $x=x_{2 n}$ and $y=w$ in (4), we have

$\rho\left(\alpha\left(x_{2 n+1}-S w\right)\right)=\rho\left(\alpha\left(T x_{2 n}-S w\right)\right) \leq \varphi\left(\rho\left(\beta\left(x_{2 n}-w\right)\right)\right)$,

therefore $\rho(\alpha(w-S w))=\lim _{n \rightarrow \infty} \rho\left(\alpha\left(x_{2 n+1}-S w\right)\right)=0$, and so $w=S w$. This completes the proof.

The following corollaries are immediate consequences of Theorem 2.1.

Corollary 2.2 Let $\mathcal{X}_{\rho}$ be a $\rho$-complete modular space. Suppose that $\varphi: \mathbb{R}^{+} \rightarrow[0, \infty)$ is an increasing and upper semicontinuous function satisfying

$\varphi(t)<t, \quad(t>0)$.

Let $C$ be a $\rho$-closed subset of $\mathcal{X}_{\rho}$ and let $T: C \rightarrow C$ be a mapping such that there exist $\alpha, \beta \in \mathbb{R}^{+}$with $\alpha>\beta$, and $\rho(\alpha(T x-T y)) \leq \varphi(\rho(\beta(x-y)))$,

for all $x, y \in C$. Then $T$ has a unique fixed point in $C$. 
Corollary 2.3 Let $\mathcal{X}_{\rho}$ be a $\rho$-complete modular space. Let $C$ be a $\rho$-closed subset of $\mathcal{X}_{\rho}$ and let $T, S: C \rightarrow C$ be mappings such that there exist $\alpha, \beta, \eta \in \mathbb{R}^{+}$with $\alpha>\beta$ and $\eta \in(0,1)$, and

$\rho(\alpha(T x-S y)) \leq \eta(\rho(\beta(x-y)))$,

for all $x, y \in C$. Then $T$ and $S$ have a unique common fixed point in $C$.

Corollary 2.4 Let $\mathcal{X}_{\rho}$ be a $\rho$-complete modular space, where $\rho$ is s-convex and satisfies the $\Delta_{2}$-condition. Let $C$ be a $\rho$-closed subset of $\mathcal{X}_{\rho}$ and let $T, S: C \rightarrow C$ be mappings such that there exist $\alpha, \beta, \eta \in \mathbb{R}^{+}$with $\alpha>\max \{\beta, \eta \beta\}$ and

$\rho(\alpha(T x-S y)) \leq \eta^{s}(\rho(\beta(x-y)))$,

for all $x, y \in C$. Then $T$ and $S$ have a unique common fixed point in $C$.

Let $\beta_{0}$ be a constant such that $\alpha>\beta_{0}>\max \{\beta, \eta \beta\}$. Then we have

$$
\begin{aligned}
\rho(\alpha(T x-S y)) & \leq \eta^{s}(\rho(\beta(x-y)))=\eta^{s}\left(\rho\left(\frac{\beta}{\beta_{0}} \beta_{0}(x-y)\right)\right) \\
& \leq\left(\frac{\beta \eta}{\beta_{0}}\right)^{s} \rho\left(\beta_{0}(x-y)\right),
\end{aligned}
$$

where $\left(\frac{\beta \eta}{\beta_{0}}\right)^{s}<1$. Hence by using Corollary 2.3 , the result follows.

\section{Acknowledgements}

The author would like to thank the editorial Board for their kindness. Moreover, the author dedicates this paper to her wife and son.

\section{References}

[1] H. Nakano, Modulared Semi-Ordered Linear Spaces, Tokyo Math. Book Ser., Vol. 1, Maruzen Co., Tokyo, 1950.

[2] J. Musielak and W. Orlicz, "On Modular Spaces", Studia Math., Vol. 18, (1959), pp.49-56.

[3] S. Koshi, T. Shimogaki, "On F-norms of quasi-modular spaces", J. Fac. Sci. Hokkaido Univ. Ser. I, Vol. 15, No. 3, (1961), pp.202-218.

[4] S. Yamamuro, "On conjugate spaces of Nakano spaces", Trans. Amer. Math. Soc., Vol. 90, (1959), pp.291-311.

[5] M.A. Krasnoselskii and Y.B. Rutickii, "Convex functions and Orlicz spaces", Fizmatgiz, Moskva [In Russian; English translation, Noordhoff, Groningen], (1961).

[6] W.M. Koslowski, Modular function spaces, Dekker: New York, Basel, (1988).

[7] J. Musielak, Orlicz Spaces and Modular Spaces, Lecture Notes in Math. Vol. 1034 , Springer-verlag, Berlin, 1983.

[8] I.D. Arandelović," on a fixed point theorem of Kirk", J. Math. Anal. Appl., Vol.301, No. 2, (2005), pp.384-385.

[9] M. Edelstein, "On fixed and periodic points under contractive mappings", J. London Math. Soc., Vol. 37, No. 1, (1962), pp.74-79.

[10] L.B Ćirić, "A generalization of Banach's contraction principle", Proc. Amer. Math. Soc., 45(2) (1974), pp.267-273.

[11] E. Rakotch, "A note on contractive mappings", Proc. Amer. Math. Soc., Vol. 13, No. 3, (1962), pp.459-465.

[12] S. Reich, "Fixed points of contractive functions", Bollettino dell'Unione Mathematica Italiana, Vol. 4, No. 5, (1972), pp. 26-42.

[13] W.A. Kirk, contraction mappings and extensions, Handbook of Metric Fixed Point Theory, W.A. Kirk and B. Sims, Eds. Kluwer Academic Publishers, Dordrecht, The Netherlands, (2001), pp.1-34. 
[14] A. Razani, E. Nabizadeh, M. Beyg Mohamadi and S. Homaei Pour, "Fixed points of nonlinear and asymptotic contraction in modular spaces", Abs. Appl. Anal., Vol. 2007, (2007), Article ID 40575,10 pages. http://www.journalofinequalitiesandapplications.com/content/2013/1/399.

[15] M. A. Khamsi, "Quasicontraction Mapping in modular spaces without $\Delta_{2}$-condition", Fixed Point Theory and Applications, Vol. 2008, (2008), Artical ID 916187, 6 pages. http://www.fixedpointtheoryandapplications.com/content/pdf/1687-1812-2008-916187.

[16] K. Kuaket and P. Kumam, "Fixed point of asymptotic pointwise contractions in modular spaces", Appl. Math. Letters, Vol. 24, (2011), pp.1795-1798.

[17] X. Wang and Y. Chen, "Fixed points of asymptotic pointwise nonexpansive mappings in modular spaces", J. Appl. Math., Vol. 2012, (2012), Article ID 319394, 6 pages. http://www.emis.de/journals/HOA/JAM/Volume2012/319394.

[18] I. Alton, M. Abbas and H. Simsek, "A fixed point theorem on cone metric spaces with new type contractivity", Banach J. Math. Anal., Vol. 5, No. 2, (2011), pp.15-24. 